\title{
FIXED POINTS OF DISK ACTIONS
}

\author{
BY ROBERT OLIVER
}

Communicated October 30, 1975

As a sequel to a previous announcement [3], the author can now give a complete classification up to homotopy type of which spaces can occur as fixed point sets of smooth actions of a given compact Lie group on disks. The result is contained in Theorems 1 to 3 below. For a group $G, G_{0}$ denotes its identity component.

THEOREM 1. Let $G$ be a compact Lie group, and $F$ a finite $C W$ complex. Then there exists a smooth action of $G$ on a disk with fixed point set having the homotopy type of $F$ if and only if:

1. $G \cong T^{n}(n \geqslant 1): F$ is Z-acyclic;

2. $G_{0}$ a torus and $\left|G / G_{0}\right|=p^{a}$ ( $p$ prime, $\left.a \geqslant 1\right): F$ is $\mathbf{Z}_{p}$-acyclic,

3. $G_{0}$ not a torus or $G / G_{0}$ not of prime power order: $\chi(F) \equiv .1\left(\bmod n_{G}\right)$ for some fixed integer $n_{G}$.

In order to describe the calculations of $n_{G}$, some classes of finite groups are defined, as in [3] and [4]. $G^{1}$ denotes the class of all $G$ with normal subgroup $P$ of prime power order, such that $G / P$ is cyclic. For $q$ prime, $G^{q}$ denotes the class of all $G$ with normal subgroup $H \in G^{1}$ of $q$-power index. Then one gets

THEOREM 2. 1. If $G_{0}$ is not a torus, then $n_{G}=1$.

2. If $G_{0}$ is a torus, then $n_{G}=n_{G / G_{0}}$.

3. If $G$ is finite, then $n_{G}=0$ if and only if $G \in G^{1}$; if $G \notin G^{1}$ then for any prime $q, q \mid n_{G}$ if and only if $G \in G^{q}$.

In Theorem 1, the necessity of the conditions in (1) and (2) follow from standard Smith theory. Sufficiency follows in (2) from Jones [2], and in (1) is trivial $(G * F$ is contractible and can be thickened up to a disk action by Theorem 6 of [4]).

For finite $G$, the existence of $n_{G}$ and the calculations in Theorem 2, part 3, were proven in [4]. Furthermore, if $G_{0}$ is a torus and $G \supseteqq G_{0}$, then $F$ clearly has the homotopy type of the fixed point set of a disk action of $G$ if and only if it does the same for $G / G_{0}$, so $n_{G}=n_{G / G_{0}}$. The case where $G_{0}$ is nontoral will be dealt with below; the above theorems say that any finite homotopy type can occur as fixed point set for such $G$.

The following result, completing the calculation of $n_{G}$, was obtained in

AMS (MOS) subject classifications (1970). Primary 57E25; Secondary 55C35. 
Theorem 4 of [5] by studying the projective obstruction $\gamma_{G}(F)$ first introduced in [4].

THEOREM 3. For any finite group $G, n_{G}=4$ if and only if:

1. $G$ is a semidirect product $0 \rightarrow Z_{n} \rightarrow G \rightarrow Z_{2} k \rightarrow 0$ ( $n$ odd) given by an automorphism $\alpha \in \operatorname{Aut}\left(\mathbf{Z}_{n}\right)$.

2. $G \notin G^{1}$, but the subgroup of index 2 is in $G^{1}$.

3. Letting $\alpha$ also denote the induced automorphism of $\mathbf{Z} \zeta_{n}$ (the ring generated by the nth roots of unity), there is no unit $u \in\left(Z_{\zeta_{n}}\right)^{*}$ such that $\alpha(u)=-u$.

Otherwise, $n_{G}$ equals 0,1 or a product of distinct primes.

Groups fulfilling conditions $1-3$ do actually exist, the smallest being given by $\left\langle a, b: a^{15}=b^{4}=e, b a b^{-1}=a^{2}\right\rangle$.

It remains to describe the case of groups with nontoral identity component; by Bredon's construction [1, $\S 1.8]$ it is enough to construct a fixed point free action of any such group on a disk. The following theorem provides some very specific examples of such actions. The concept of a family of subgroups is used, as defined by tom Dieck.

THEOREM 4. Let $G$ be a compact Lie group, and $F$ a nonempty family of subgroups. Then there exists a smooth action of $G$ on a disk $D$ such that $D^{H}$ is a disk for $H \in F$ and empty for $H \notin F$, if and only if:

1. For any pair of subgroups $H \triangleleft K$ in $G$, for which $K / H$ has prime order, either both $H$ and $K$ are in $F$ or neither is.

2. Fis closed in the space of closed subgroups of $G$ with the Hausdorff topology.

In particular, the family of subgroups $H$ such that $H_{0}$ is a torus and $H / H_{0}$ solvable meets these conditions.

\section{REFERENCES}

1. G. Bredon, Introduction to compact transformation groups, Academic Press, New York, 1972.

2. L. Jones, The converse to the fixed point theorem of P. A. Smith. I, Ann. of

Math. (2) 94 (1971), 52-68. MR 45 \#4427.

3. R. Oliver, Fixed points of finite group actions on contractible complexes, Bull. Amer. Math. Soc. 81 (1975), 482-484.

4. - Fixed point sets of group actions on finite acyclic complexes, Comment Math. Helv. 50 (1975), 155-177.

5. - Projective obstructions to group actions on disks (to appear)

MATEMATISK INSTITUT, ÅRHUS UNIVERSITET, ÅRHUS, DENMARK 